\title{
Etiology of eating disorders explored as patience for a cure thins
}

Headlines about skeletal supermodels have fueled the perception that eating disorders are caused by cultural pressure to be thin. In tandem, treatments for such disorders have long centered on psychotherapy, often focused on improving patients' body image.

But new studies have now started plumbing the biological sources of anorexia and bulimia nervosa - and also offering hints as to how current and future drugs might target that those pathways.

In February, researchers initiated the largest multicenter study funded by the National Institute of Mental Health (NIMH) for the treatment of anorexia. The clinical trial will compare two different forms of psychotherapy in combination with either the antidepressant Prozac or a placebo. The month before, Canadian investigators at the Children's Hospital of Eastern Ontario

을 Research Institute in Ottawa launched the first randomized clinical trial testing the antipsychotic drug olanzapine in subjects with anorexia. Case studies indicate that

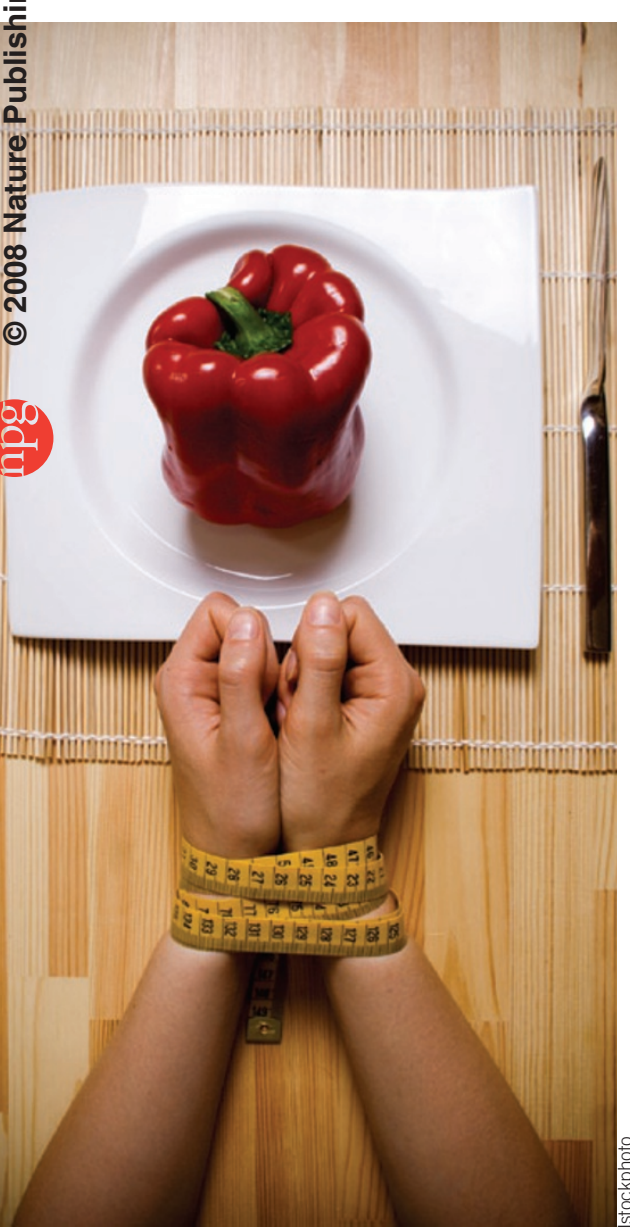

Food matters: Genes linked to eating disorders olanzapine promotes weight gain and reduces hyperactivity.

The trials mark the beginning of a revolution in thinking about the etiology of eating disorders, according to experts. "We had been saying there are no medications for anorexia nervosa," says psychologist Cindy Bulik, at the University of North Carolina at Chapel Hill. "But truth be told, that, in part, is the byproduct of lagging biological research."

Whereas genome studies have uncovered a multitude of culprits in other mental illnesses (such as depression), anorexia and bulimia have taken a back seat. "Eating disorders are not seen as high priority kinds of illness for pharmaceutical companies," says James Mitchell, president and scientific director of the Neuropsychiatric Research Institute in Fargo, North Dakota.

Some experts believe that the exploration of drug targets for eating disorders has moved slowly in part because the illnesses are relatively rare. By some estimates, anorexia afflicts only $0.5-3.4 \%$ of females. Moreover, the mortality rate of the disorder reaches as high as $20 \%$ (Int. J. Eat. Disord. 38, 143146; 2005; N. Engl. J. Med. 340, 1092-1098; 1999). Bulimia, with its $1.1-4.2 \%$ incidence, offers an only slightly larger market for medications.

But, as organizations such as the NIMH are stepping in to provide funding, researchers have started uncovering genes that were never on the list of suspects for these disorders. At the same time, neuroimaging tools have hinted that faulty brain circuitry might cause the distorted thoughts and problems involved in obsessionality, perfectionism, anxiety and depression-conditions that go hand-in-hand with anorexia and bulimia. Those symptoms, too, might be treated with medication.

For example, Walter Kaye of the University of California, San Diego has performed brain mapping studies of women with histories of anorexia and bulimia. Kaye has pinpointed neurobiological vulnerabilities in the serotonin system, including receptors in brain regions responsible for processing reward. In addition, genetic mapping studies have linked eating disorders to variations in serotonin receptors, one of the dopamine receptors and a neurotransmitter called brain-derived neurotropic factor, which has a general role in the growth and maintenance of nerve cells.

The next step is to try matching these new molecular discoveries to treatments. Farthest along in this approach is bulimia. Antidepressant medications, such as selective serotonin reuptake inhibitors (SSRIs), have shown evidence of therapeutic value in bulimia. In fact, the US Food and Drug Administration approved one such drug, Prozac, to treat bulimia.

\section{Action plan}

Experts say the best course of action for bulimia is to try a kind of psychotherapy called cognitive behavioral therapy first and then introduce antidepressants-if psychotherapy alone isn't working or if a patient is showing clinical signs of depression as well as bulimic behaviors.

For anorexia, researchers have started exploring the efficacy of peptide hormones, including compounds that mimic ghrelin (a gut hormone that stimulates appetite) and growth hormone. Investigators have also turned their attention to the potential of molecules that inhibit neurotransmitter receptors called $\mathrm{Y} 1$ and $\mathrm{Y} 5$, involved in feeding, and those molecules that block cell surface receptors for orexin, a hormone that influences sleep and appetite.

Bulik says that clinicians often prescribe SSRIs to patients with anorexia. But studies show that the drugs won't work when the person is at an extremely low weight. This might result from the fact that the body synthesizes serotonin from the amino acid tryptophan, which is obtained through a normal diet.

Another clinical practice, says Bulik, is putting individuals with eating disorders on birth control to regulate periods and prevent osteoporosis. She points to a lack of evidence to supporting this treatment: "There is absolutely no data for that," Bulik says. "Thus, we are asking clinicians to hold off."

The study of drugs for treating eating disorders is very much "in its infancy," according to Bulik, which leads to a large discrepancy between early insights obtained from basic research and what physicians and therapists actually do in practice. "When a person is less than $85 \%$ of ideal weight, of course, flags should go up all over the place," says psychologist Robinson Welch, at Washington University in St. Louis, Missouri, one of the co-investigators of the NIMHfunded, multicenter anorexia trial. "But when the underlying issue is an eating disorder, we're asking for caution before beginning medication."

Trisha Gura, Boston 